\title{
A microfluidic device with integrated sonication and immunoprecipitation for sensitive epigenetic assays
}

Zhenning $\mathrm{Cao}^{\dagger}$ and Chang $\mathrm{Lu}^{\mathrm{II}, *}$

${ }^{\dagger}$ Department of Biomedical Engineering and Mechanics, Virginia Tech, Blacksburg, VA 24061, USA

${ }^{\text {II }}$ Department of Chemical Engineering, Virginia Tech, Blacksburg, VA 24061, USA

Supporting Information: Table S1-S2 and Video S1-S4 
Table S1. Primer sequences used in ChIP-qPCR.

\begin{tabular}{l|l|l}
\hline \multirow{2}{*}{ C9orf3 } & \multicolumn{2}{l}{ GM12878 and H3K4me3 } \\
\cline { 2 - 3 } & R & CCTCCTCAGTTCTCCCAGACT \\
\hline \multirow{2}{*}{ UNKL } & F & CAGCCAGGCAGACACCTAGGTGTGAC \\
\cline { 2 - 3 } & R & TCCTATGGCTCCCCAGGT \\
\hline \multirow{2}{*}{ 1 } & F & TCATCTGCAAATGGGGACAA \\
\cline { 2 - 3 } & R & AGGACACCCCCTCTCAACAC \\
\hline \multirow{2}{*}{ N2 } & F & ATGGTTGCCACTGGGGATCT \\
\cline { 2 - 3 } & R & TGCCAAAGCCTAGGGGAAGA \\
\hline
\end{tabular}

Table S2. Primer sequences used in MeDIP-qPCR.

\begin{tabular}{l|l|l}
\hline \multirow{2}{*}{ SNRPN } & \multicolumn{3}{l}{ gDNA of GM12878 and 5-mC } \\
\cline { 2 - 3 } & $\mathrm{R}$ & GGCTCAACACCCCCTAAATA \\
\hline \multirow{2}{*}{ MAGEA1 } & $\mathrm{F}$ & GTTCCAGGTGGGTACATCAAAAACATC \\
\cline { 2 - 3 } & $\mathrm{R}$ & GAACTCTACGCCGTCCCTCAG \\
\hline \multirow{2}{*}{ GABRB3 } & $\mathrm{F}$ & CCTGCAACTTTACTGAATTTAGC \\
\cline { 2 - 3 } & $\mathrm{R}$ & GGAATCTCACTTTCACCACTGG \\
\hline \multirow{2}{*}{ GAPDH } & $\mathrm{F}$ & CGTAGCTCAGGCCTCAAGAC \\
\cline { 2 - 3 } & $\mathrm{R}$ & GCTGCGGGCTCAATTTATAG \\
\hline
\end{tabular}

\section{Supplementary Videos}

Video S1: Cavitation at the edge of a microfluidic chamber without crescent structures. Sinewave $\mathrm{AC}$ with $\mathrm{V}_{\text {peak }}$ of $20 \mathrm{~V}$ and $61 \mathrm{kHz}$ was applied. The video was captured and played at a frame rate of $8 \mathrm{fps}$.

Video S2: Cavitation in a microfluidic chamber containing multiple crescent shapes. $\mathrm{V}_{\text {peak }}$ was gradually increased from 10 to $25 \mathrm{~V}$. Sine-wave at $61 \mathrm{kHz}$ was applied. The video was captured at a frame rate of $8 \mathrm{fps}$ and is played at $20 \mathrm{fps}$.

Video S3: Acoustic streaming around a crescent shape. Sine-wave of $61 \mathrm{kHz}$ and $\mathrm{V}_{\text {peak }}$ of $10 \mathrm{~V}$ was applied. 10X objective was used. The video was captured and played at a frame rate of $8 \mathrm{fps}$.

Video S4: A close look of acoustic streaming around the tip of a crescent shape. Sine-wave of 61 $\mathrm{kHz}$ and $\mathrm{V}_{\text {peak }}$ of $10 \mathrm{~V}$ was applied. 20X objective was used. The video was captured and played at a frame rate of $8 \mathrm{fps}$. 